\title{
VASCULAR LOOP AS A CAUSE OF INCAPACITATING DIZZINESS
}

\author{
Brian F. McCabe, M. D. and Bruce J. Gantz, M. D.
}

Vascular compression of cranial nerves has been accepted as the cause of tic doloreaux, hemifacial spasm and glossopharyngeal neuralgia ${ }^{1 \sim 6)}$. There are reports that vertigo, hearing loss and tinnitus also are the result of vascular loops against the eighth cranial nerve ${ }^{6 \sim 10)}$.

Existing reports of eighth nerve vascular compression have lacked a careful otologic workup. Few of the patients reported as "improved" had adequate audiometric evaluation or vestibular function tetsts in these reports. The one exception is a small group of patients studied by Applebaum and Valvassori ${ }^{11)}$. The presenting complaint of that group was unilateral hearing loss. With further investigation, episodes of vertigo were noted in about half of the subjects. The authors found the vascular anomalies on air cisternogram-computed tomography (CT) obtained to rule out an acoustic tumor. However, surgical decompression of the eighth nerve was not performed on any of the patients, so proof that there was an association between the vascular loop and the symptoms is lacking.

The senior author reported five years ago a small group of patients felt to have vascular compression as the etiology of their vertigo ${ }^{12)}$. Eight patients were found to have a vascular loop against (and even indenting) the vestibular nerve during middle cranial fossa vestibular neurectomy. All of the patients had relief of symptoms after the nerve section. Sectioning the nerve, however, failed to define that the symptoms were due to the loop or inner ear disease, except in two of the seven patients who had no inner ear function remaining (no hearing and no response

Division of Otology-Neurotology Department of Otolaryngology-Head and Neck Surgery, The University of Iowa, Iowa City, Iowa to ice water calorics), whose symptoms could not have been mediated from the endorgan. These two patients had operations on their labyrinths proir to being seen by us which destroyed inner ear function. Justifiably neurotologists have been skeptical of the causal relationship of vascular loop compression and vestibular nerve symptoms. Reasons for this skepticism include vague patient histories, inadequate workup and follow-up data and, particularly, existing anatomic studies demonstrating vascular structures crossing the cerebellopontine cistern in most anatomic specimens ${ }^{13)}$.

We describe here a group of patients with intractable and disabling motion intolerance and vertigo who have undergone vascular decompression of the vestibular nerve. Meticulous documentation of symptoms, laboratory studies, and results indicate that a vascular loop syndrome must be considerered in the differential diagnosis of patients with distressing and finally incapacitating vestibular dysfunction. Moreover, we believe that there is within the history of patients here described, a symptom-set which identifies those patients deserving studies to image a possible vestibular nerve vascular loop.

\section{Meterials and Methods}

The records of 34 patients having undergone vascular loop decompression of the vestibular nerve were reviewed. Twenty-nine patients have been followed from six months to five years and are included in this retrospective analysis. Patient records, a questionnnaire, and telephone interviews were used to obtain follow-up data.

Dizziness was the presenting complaint in all patients. The average duration of symptoms was 6.4 years (range : 9 months to 19 years). The preponderance of patients was in the 20 to 40 year age 
group. Eleven patients were male and 18 female.

The predominant symptom and the major reason for seeking medical attention in 27 of the 29 was disabling motion intolerance. Quick movement, repetitive head turning and/or visual observation of movement precipitated unsteadiness and, in many instances, nausea and vomiting. Many of the patients had stopped driving because of the symptoms. All patients described vertigo with spells lasting seconds to minutes especially early in their disease.

A previous diagnosis of Meniere's disease had been made in 15 of 29 (52\%), and almost all (14) were thought to have vestibular Meniere's disease. All had failed medical management for vestibular Meniere's disease, and in two of two, and endolymphatic shunt did not alter their symptoms. In most of these patients the spells had become more frequent and lasted only a few minutes. The usual history was weekly to daily balance disturbances. Growing motion intolerance became the increasingly dominant symptom.

The patients with a vascular loop receiving a diagnosis of vestibular Meniere's disease had a rather typical pattern. Early in their disease they had seizures of vertigo (without auditory symptoms) and were normal between spells. As the months or years ensued, there appeared motion intolerance between spells, making the diagnosis of vestibular Meniere's disease suspect. As time progressed further, the spells became less severe and further apart and, at the same time, the motion intolerance grew, to become the main problem. The latter symptom became incapacitating.

Additional complaints included two patients with unilateral hearing loss, four with unilateral tinnitus, and one exhibiting hemifacial spasm. Many could describe the offending ear because of aural fullness or other vague symptoms. Stress aggravated their symtpoms. The motion intolerance finally had interfered with their daily routine to the point that they were incapacitated, and this was the point which led to their acceptance of decompression via craniotomy. Audiometric evaluation demonstrated normal or symmetric hearing in all but two who had been operated upon. Speech discrimination was normal in the other 27, and none displayed abnormal acou- stic reflex decay.

Electronystagmographic examination revealed in $86 \%(25 / 29)$ of subjects. Canal paresis was present on the side of the vascular compression in $13 / 25$ $(52 \%)$. Seven (28\%) patients displayed eyes closed spontaneous nystagmus. The direction of the nystagmus was variable. Positional nystagmus was observed in five $(20 \%)$, and a dysrhythmic pattern of the nystagmus following caloric stimulation (a

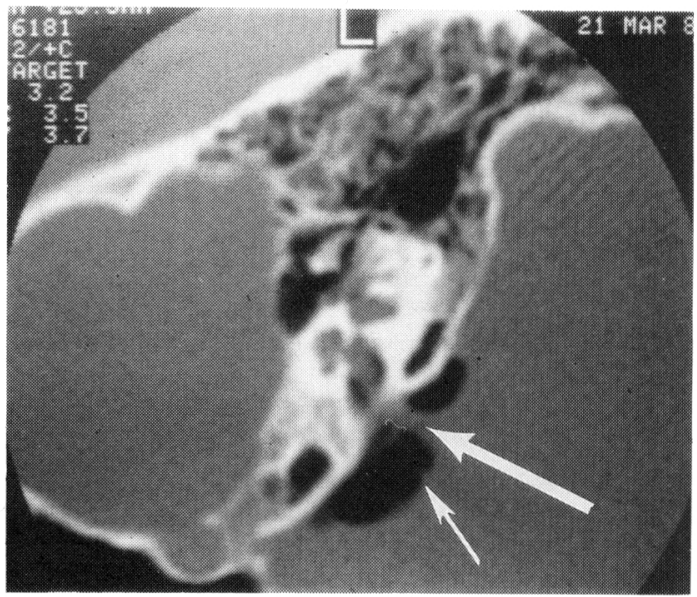

Figure 1: Air cisternogram. The large arrow points to the VII-VIII nerve complex made visible by air contrast, and the small arrow to the vescular loop curving towards the neurobundle and contacting it and then curving away from it posteriorly.

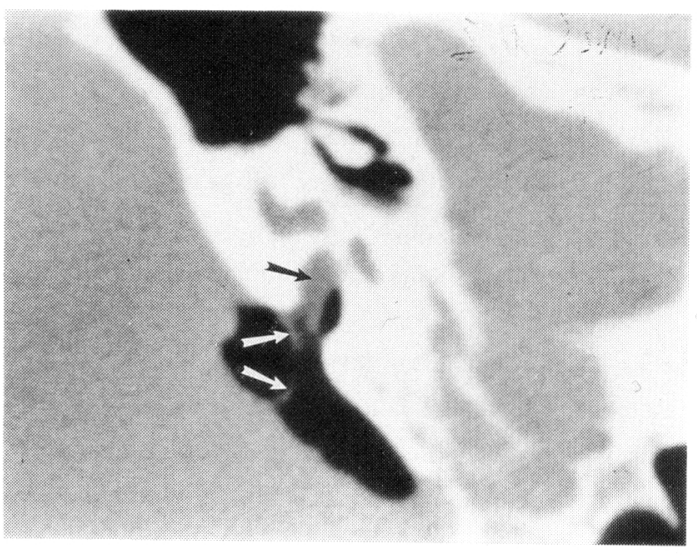

Figure 2: Air cirsternogram. The black arrow points to the VII-VIII nerve complex and the white arrows to the vascular loop. 
varying amplitude envelope) was seen in five (20\%) tracings.

Diagnosis of a vessel in contact with the vestibular nerve was made by obtaining an air cisternogram CT examination of both cerebellopontine angles. The air study visualized the nerve bundle and a nonionic iodinated intravenous contrast material was injected to highlight vascular structures. A vascular structure contacting the neurovascular bundle was demonstrated in all 29 patients (Fig. 1,2). Three of the patients displayed bilateral vascular loops. The side decompressed in these three was based on the patient's signs and symptoms of laterality and the closeness of contact of the vessel and nerve on CT. The contact point varied from the root outlet zone to mid-internal auditory canal.

Three different surgical approaches were employed to decompress the eighth nerve in this series. Initially the middle cranial fossa $(\mathrm{N}=6)$ and retrolabyrinthine $(\mathrm{N}=10)$ exposures were used. Selection of the route was determined by the point of contact of the vessel and nerve on air CT study. The preferred approach at this time is a limited retrosigmoid craniotomy $(\mathrm{N}=13)$. Exceptions are cases where the contact appears to be very lateral in the IAC, where the middle cranial fossa exposure would provide better access.

Intraoperatively, all patients had one or more vessels in contact with the vestibular portion of the eighth nerve. In most, the offending vessel was either the anterior inferior cerebellar artery (AICA), the posterior inferior cerebellar artery (PICA), or branches of them. Some vessels indented the nerve, while some passed between CN VII and VIII. the vessels are mobilized away from the nerve with blunt dissection. Occasionally, thickened arachnoid required sharp dissection. Small pieces of tufted teflon felt were then placed between the nerve and vessels to mainain the decompressive position of the offending $\operatorname{vessel}(s)$.

\section{Results}

Overall results in this series have been very gratifying. All but one patient has benefited from surgical decompression. Twenty-three patients (79\%) have relief of their vertigo and motion intolerance, and are back to their daily routine. Five patients (17
\%) are improved, but continue to experience some symptoms when they "overdo", or are fatigued. One of the five is relieved, but not yet back to his daily routine, and three are considered partial failures, with only $50 \%$ resolution of symptoms by patients estimate. The one patient considered to be a failure states that the operation did not alter his symptoms. He also failed a prior endolymphatic shunt and medical management for vestibular Meniere's disease. Preoperative workup revealed symmetric hearing, and a canal paresis on the side of the vessel. This patient was one of our earliest in the program, and an insufficient amount of teflon felt may have been placed or the felt's position may have shifted. He continues to have motion intolerance and spells of vertigo. Of the partial failure group, each was asked on successive outpatient follow-up visits if they had it to do again, would they have the operation. Each said "yes".

Many patients with aural fullness describe a remission in the immediate postoperative period, which is sustained. The motion intolerance symptoms gradually improve over a period of weeks $(\mathrm{N}=9)$ to onethree months $(\mathrm{N}=17)$. Two patients experienced improvement at as late as four and five months before, as they state, they "turned the corner." We assume latency of recovery to be due to the period of time for remyelination to occur. Demyelination of the peripheral portion of the nerve has been shown in hemifacial spasm, tic doloreaux, and essential blepharospasm, and it is reasonable to expect in this disease also since the mechanism is the same. A prolonged recovery period has also been seen in patients following vascular decompression for hemifacial spasm.

No patients complained of worsened symptomatology and no patients have experienced return of symptoms following improvement.

Relief of the other symptoms has been variable. Two of four patients state their tinnitus is improved. Hemifacial spasm in the one patient with it has been alleviated. The preoperative hearing loss in one patient is unchanged.

The surgical complications in this series have been minimal. Two patients ( $\mathrm{MCF} \mathrm{N}=1$, retrolab $\mathrm{N}=1$ ) experienced cerebrospinal fluid rhinorrhea in the im- 
mediate postoperative period. Both leaks were controlled with a temporary lumbar spinal drain and the leaks ceased prior to discharge. One patient complained of diplopia two weeks after discharge from the hospital. Examination revealed bilateral CN VI paresis and papilledema. A diagnosis of pseudo-tumor cerebri was made, and the symptoms cleared following treatment with steroids. The exact cause of pseudo-tumor cerebri is unknown; it could have been secondary to compression of the sigmoid sinus during the retrolabyrinthine exposure. Severe and prolonged occipital headache requiring medication has occurred in three cases, beginning two-three months postoperatively. It has occurred with each of the three surgical approaches. There has been no hearing loss, facial nerve paralysis, meningitis, intracranial hemorrhage or death in this seres.

\section{Discussion}

The patient's history continues to be the single important clue to the diagnosis of the dizzy patient. These vascular loop patients are probably the most illustrative of that rule. They look well, act well, have seen many doctors for their problem, and have received little understanding and no daignosis. Many have received psychotherapy without benefit. All have received meclizine, an ineffective drug in vestibular disease except for prevention of motion sickness, without benefit. Many patients however fail to fit established criteria of peripheral vestibular dysfunction, and are labeled as a "central" problem or atypical peripheral dysfunction. The patients in this series fit into this nondescript latter group. The patient histories were consistent with verrigo of peripheral origin, and the ENG studies supported a peripheral cause. The list of disorders which present with symptoms of short vertiginous spells with interim motion intolerance continues to expand. Included are perilymph fistula, autoimmune inner ear disease, acoustic neuroma, and vascular compression syndrome.

Vascular compression syndrome should be considered when patients describe frequent brief spells of vertigo, disabling motion intolerance, failure of multiple medical and surgical regimens, normal or symmetric hearing, and evidence of peripheral vestibular dysfunction on ENG testing. Many in this se- ries had been diagnosed as having vestibular Meniere's disease, but uniformly obtained no relief with usual medical regimen of diruetics and $1500 \mathrm{mg}$ salt diet. Centrally acting drugs such as Tegretol, Dilantin, and Klonopin were not helpful, and propanolol for vestibular migraine provided no relief in one patient. Psychiatric evaluation had been obtained in a few patients to identify a functional etiology for their symptoms, and treatment was without success in any. Universally, these patients were severely distressed and unable to carry out their daily routines.

The decision to advise the patient to undergo a vascular decompression operation in this series was based on history, ENG findings, and imaging of a vessel contacting the eighth nerve on air CT study. Four additional patients not included in this series presented with similar findings, but had negative air CT studies. They have not been operated upon, and are being treated with various central drug regimens. Three of the patients continue to have symptoms.

This is the only known series wherein the decision for decompression has been based on imaging studies. Other series ${ }^{1 \sim 10)}$ of trigeminal neuralgia and hemifacial spasm have not relied on imaging procedures to confirm the diagnosis. We recommend that air CT imaging be performed on all patients suspected of having vestibular dysfunction due to vascular compression syndrome.

There is considerable skepticism that vascular compression induces vestibular dysfunction. One argument against the disorder is that anatomic studies commonly describe vessels traversing the cerebellopontine cistern. Mazzoni ${ }^{14)}$ identified loops of AICA within the ICA in $67 \%$ of specimens, while Sunderland $^{15)}$ observed the finding $40 \%$ of the time. Applebaum and Valvassori ${ }^{11)}$, on the other hand reported evidence of aberrant vessels contacting the eighth cranial nerve in only $9 \%(20 / 287)$ of pneumoCT studies. The vessels were unilateal in their series and always on the side of vestibular dysfunction. Prominent vascular loops are also a rare finding in our series of air CT scans performed on patients suspected of having acoustic tumors. The discrepancy between fixed anatomic specimens and live patients may be due to many factors. Valvassori's explanation* is that vessels free in the $\mathrm{CP}$ angle are 
mobile. The dwell time of the CT scanner is such that mobile pulsating vessels blur and are not visualized, whereas vessels contacting or fixed to nerves do not blur. It should also be noted that not all vessels contacting nerves cause symptoms. We found large vessels passing between and contacting both $\mathrm{CN}$ VII and VIII in over $20 \%$ of the cases and only one case was there hemifacial spasm. Apparently, the character and degree of contact of the vessel and nerve is important in the demyelination of the nerve and mediation of symptoms.

The actual injury caused by the offending vessel is suspected to be a demylination secondary to pulsatile compression. This phenomenon may occur anywhere along the nerve. It is proposed that the most likely site of injury is at the transition zone between the central glial and peripheral myelinated segment. The myelin is very thin at this junction, and compression may cause myelin loss with resultant neuronal cross-talk. The transition zone, or pial ring, is found close to the root outlet zone of $\mathrm{CN} \mathrm{V}$ and V.II. The root outlet zone is the most common site of compression reported in trigeminal neuralgia and hemifacial spasm. CN VIII has a long glial segment and the transition zone in some ears exten'ds well into the internal auditory canal. These anatomic differences may account for the observation that not all vessels contacting the cranial nerves produce symptoms.

The results of the present series support the concept that aberrant vescular compression can induce severe vestibular dysfunction. Separation of the vessels and nerve alleviated the vestibular symptoms in $79 \%$ of patients, and improved $97 \%$ of this population. In our hands, the retrosigmoid approach offers the best visibility and working space for decompression. Dural closure is more easily accomplished with this procedure, and should result in few CSF leaks.

Decompression of the nerve, in contrast to sectioning, also avoids the morbidity associated with vestibular nerve section requiring the usual period of central compensation. There is no postoperative vestibular crisis. Patients leave the hospital soon after the wound is stable, and no patients have the decrescendo unsteadiness and ataxia that usually follows nerve section. Vascular decompression does not appear

Equilibrium Res Vol. 48(2) to induce further injury, since none of our patients experienced an increase in symptoms, hearing loss, or facial paralysis. It is important to recognize and image patients with a possible vascular compression syndrome, because the patient may be in that group offered a vestibular neurectomy due to the severity of their disability.

In summary, vascular compression syndrome appears to be another cause of severe vestibular dysfunction, and must be considered in the differential diagnosis of patients presenting with an unremitting pattern of peripheral vertigo and motion intolerance. Vascular compression syndrome patients exhibit brief frequent spells of vertigo, disabling motion intolerance precipitated by both head movement, and visual precipitation of symptoms. They will most likely have evidence of peripheral vestibular dysfunction on ENG. Air CT scan with I. V. contrast can confirm the presence of a compressing vessel. Appropriate decompression of $\mathrm{CN}$ VIII can result in the alleviation or elimination of the incapacitating symptoms in almost all patients.

\section{Acknowledgments}

The authors thank Dr. Kenneth Dolan for his interest and expertise in the interpretation of the air CT studies, and also Dr. Galdino Valvassori for his help in the same in the early patients in this series.

\section{REFERENCES}

1) Dandy WE : Concerning the cause of trigeminal neuralgia. AM J Surg $24: 447-455,1932$

2) Gardner WS : Concerning the mechanism of trigeminal neuralgia and hemifacial spasm. J. Neurosurg $19: 947-958,1962$

3) Jannetta PJ : Arterial compression of the trigeminal nerve at the pons in patients with trigeminal neuralgia. J Neurosurg (suppl) $26: 159$ 162,1967

4) Jannetta PJ : Neurovascular compression of the facial nerve in hemifacial spasm by microsurgical technique. In Reconstructive Surgery of the Brain Arteries, edited by RT Merei, pp 193199, Akademiai Kiado, Budapest, Hungary, 1974

5 ) Jannetta PJ, Abbasy M, Maroon JG, Ramor FM, Albin MS : Etiology and definitive microsurgical treatment of hemifacial spasm : operative techni- 
ques and results in 47 patients. J Neurosurg 47 : 321-328, 1977

6) Jannetta PJ : Observation on the etiology of trigeminal neuralgia, hemifacial spasm, acoustic nerve dysfunction and glossopharyngeal neuralgia. Difinitive microsurgical treatment and results in 117 patients. Neurochirurgia (Stuttg) 20:145154, 1977

7) Jannetta PJ : Neurovascular cross compression in patients with hyperactive dysfunction symptoms of the eighth cranial nerve. Surg Forum $26: 467-469,1975$

8 ) Jannetta PJ : Neurovascular cross-compression of the eighth cranial nerve in patients with vertigo and tinnitus. In The Cranial Nerves, ed. Samii M, Jannetta PJ, pp 552-555, Springer-Verlag, Berlin, 1981

9) Bertrand RA, Molina P, Hardy J: Vestibular syndrome and vascular anomaly in the cerebellopontine angle. Acta Otolaryngol (Stockh) 83 : 187-194, 1977
10) Leclercq TA, Hill CL, Grisoli F : Retrosigmoid microsurgical approach to vascular compression of the eighth cranial nerve. Laryngoscope 90 : 1011-1017, 1980

11) Applebaum EL, Valvassori $G$ : Internal auditory canal vascular loops: audiometric and vestibular system findings. J Otol Nov (suppl) $6: 110$ 113, 1985

12) McCabe BF, Harker LA : Vascular loop as a cause of vertigo. Ann Otol, Rhinol, Laryngol, $92: 542-543,1983$

13) Quaking GE : Microsurgical anatomy of the arterial loops in the ponto-cerebellar angle and the internal auditory meatus. In The Cranial Nerves. ed. Samii M, Jannetta PJ, pp 378-390, Springer-Verlag, Berlin, 1981

14) Mazzoni A, Hansen $C C$ : Surgical anatomy of the arteries of the internal auditory canal. Arch Otolaryngol $91: 128-135,1970$

15) Sunderland $S:$ The arterial relations of the internal auditory meatus. Brain $68: 23-27,1945$ 